\title{
Constructive Proof of the Existence of Nash Equilibrium in a Finite Strategic Game with Sequentially Locally Nonconstant Payoff Functions
}

\author{
Yasuhito Tanaka \\ Faculty of Economics, Doshisha University, Kamigyo-ku, Kyoto 602-8580, Japan \\ Correspondence should be addressed to Yasuhito Tanaka, yasuhito@mail.doshisha.ac.jp \\ Received 5 August 2011; Accepted 19 September 2011 \\ Academic Editor: T. Karakasidis
}

Copyright () 2012 Yasuhito Tanaka. This is an open access article distributed under the Creative Commons Attribution License, which permits unrestricted use, distribution, and reproduction in any medium, provided the original work is properly cited.

\begin{abstract}
We will constructively prove the existence of a Nash equilibrium in a finite strategic game with sequentially locally nonconstant payoff functions. The proof is based on the existence of approximate Nash equilibria which is proved by Sperner's lemma. We follow the Bishop-style constructive mathematics.
\end{abstract}

\section{Introduction}

It is often said that Brouwer's fixed-point theorem cannot be constructively proved.

Refernce [1] provided a constructive proof of Brouwer's fixed-point theorem. But it is not constructive from the viewpoint of constructive mathematics á la Bishop. It is sufficient to say that one-dimensional case of Brouwer's fixedpoint theorem, that is, the intermediate value theorem, is nonconstructive. See [2] or [3]. On the other hand, in [4] Orevkov constructed a computably coded continuous function $f$ from the unit square to itself, which is defined at each computable point of the square, such that $f$ has no computable fixed point. His map consists of a retract of the computable elements of the square to its boundary followed by a rotation of the boundary of the square. As pointed out by Hirst in [5], since there is no retract of the square to its boundary, Orevkov's map does not have a total extension.

The existence of a Nash equilibrium in a finite strategic game also cannot be constructively proved. Sperner's lemma which is used to prove Brouwer's theorem, however, can be constructively proved. Some authors have presented a constructive (or an approximate) version of Brouwer's theorem using Sperner's lemma. See [3, 6]. Thus, Brouwer's fixed-point theorem can be constructively proved in its constructive version. Also van Dalen in [3] states a conjecture that a uniformly continuous function $f$ from a simplex to itself, with property that each open set contains a point $x$ such that $x \neq f(x)$, which means $|x-f(x)|>0$, and also at every point $x$ on the boundaries of the simplex $x \neq f(x)$, has an exact fixed point. We call such a property of functions local nonconstancy. Further, we define a stronger property sequential local nonconstancy. In another paper [7], we have constructively proved Dalen's conjecture with sequential local nonconstancy.

In this paper, we present a proof of the existence of a Nash equilibrium in a finite strategic game with sequentially locally nonconstant payoff functions. In the next section, we present Sperner's lemma for an $n$-dimensional simplex whose constructive proof is omitted indicating references. In Section 3, we present a proof of the existence of a Nash equilibrium in a finite strategic game with sequentially locally nonconstant payoff functions. The proof is based on the existence of approximate Nash equilibria which is proved by Sperner's lemma. We follow the Bishop-style constructive mathematics according to $[2,8,9]$.

\section{Sperner's Lemma}

Let $\Delta$ denote an $n$-dimensional simplex. $n$ is a finite natural number. For example, a 2-dimensional simplex is a triangle. Let us partition or triangulate the simplex. Figure 1 is an example of partitioning (triangulation) a 2-dimensional 


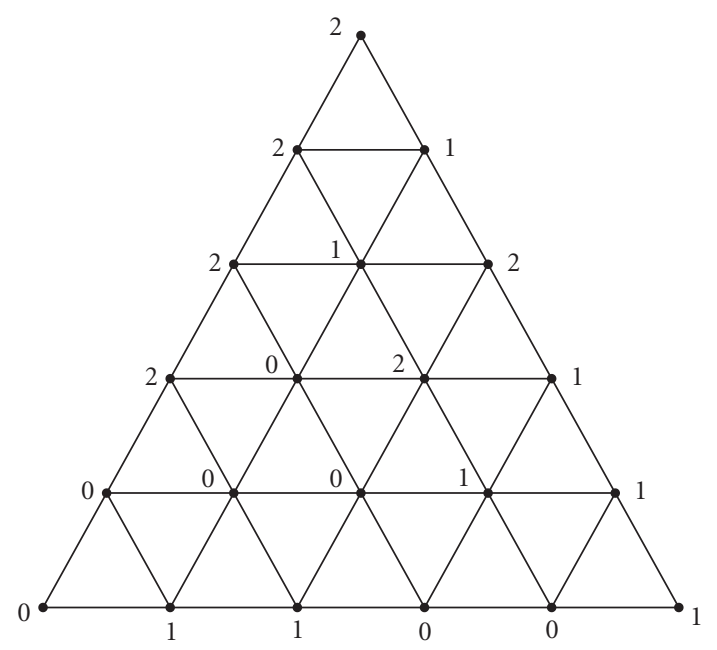

FIgURE 1: Partition and labeling of 2-dimensional simplex.

simplex. In a 2-dimensional case, we divide each side of $\Delta$ in $m$ equal segments and draw the lines parallel to the sides of $\Delta . m$ is a finite natural number. Then, the 2-dimensional simplex is partitioned into $\mathrm{m}^{2}$ triangles. We consider partition of $\Delta$ inductively for cases of higher dimension. In a 3-dimensional case, each face of $\Delta$ is a 2-dimensional simplex, and so it is partitioned into $\mathrm{m}^{2}$ triangles in the above-mentioned way, and draw the planes parallel to the faces of $\Delta$. Then, the 3-dimensional simplex is partitioned into $\mathrm{m}^{3}$ trigonal pyramids. And this is similar to cases of higher dimension.

Let $K$ denote the set of small $n$-dimensional simplices of $\Delta$ constructed by partition. Vertices of these small simplices of $K$ are labeled with the numbers $0,1,2, \ldots, n$ subject to the following rules.

(1) The vertices of $\Delta$ are, respectively, labeled with 0 to $n$. We label a point $(1,0, \ldots, 0)$ with 0 , a point $(0,1,0, \ldots, 0)$ with 1 , a point $(0,0,1 \ldots, 0)$ with $2, \ldots$, and a point $(0, \ldots, 0,1)$ with $n$. That is, a vertex whose $k$ th coordinate $(k=0,1, \ldots, n)$ is 1 and all other coordinates are 0 is labeled with $k$.

(2) If a vertex of $K$ is contained in an $n$-1-dimensional face of $\Delta$, then this vertex is labeled with some number which is the same as the number of one of the vertices of that face.

(3) If a vertex of $K$ is contained in an $n$-2-dimensional face of $\Delta$, then this vertex is labeled with some number which is the same as the number of one of the vertices of that face, and so on for cases of lower dimension.

(4) A vertex contained inside of $\Delta$ is labeled with an arbitrary number among $0,1, \ldots, n$.

A small simplex of $K$ which is labeled with the numbers $0,1, \ldots, n$ is called a fully labeled simplex. Sperner's lemma is stated as follows.
Lemma 1 (Sperner's lemma). If one labels the vertices of $K$ following the rules (1) (4), then there are an odd number of fully labeled simplices, and so there exists at least one fully labeled simplex.

Proof. About constructive proofs of Sperner's lemma, see [10] or [11].

Since $n$ and partition of $\Delta$ are finite, the number of small simplices constructed by partition is also finite. Thus, we can constructively find a fully labeled $n$-dimensional simplex of $K$ through finite steps.

\section{Nash Equilibrium in Strategic Game}

Let $\mathbf{p}=\left(\mathbf{p}_{0}, \mathbf{p}_{1}, \ldots, \mathbf{p}_{n}\right)$ be a point in an $n$-dimensional simplex $\Delta$, and consider a function $\varphi$ from $\Delta$ to itself. Denote the $i$ th components of $\mathbf{p}$ and $\varphi(\mathbf{p})$ by $\mathbf{p}_{i}$ and $\varphi_{i}(\mathbf{p})$ or $\varphi_{i}$.

The definition of local nonconstancy of functions is as follows.

Definition 2 (local nonconstancy of functions). (1) At a point $\mathbf{p}$ on the faces (boundaries) of a simplex $\varphi(\mathbf{p}) \neq \mathbf{p}$, this means that $\varphi_{i}(\mathbf{p})>\mathbf{p}_{i}$ or $\varphi_{i}(\mathbf{p})<\mathbf{p}_{i}$ for at least one $i$.

(2) In any open set in $\Delta$, there exists a point $\mathbf{p}$ such that $\varphi(\mathbf{p}) \neq \mathbf{p}$.

Next, by reference to the notion of sequentially at most one maximum in [12], we define the property of sequential local nonconstancy.

First, we recapitulate the compactness (total boundedness with completeness) of a set in constructive mathematics. $\Delta$ is compact in the sense that for each $\varepsilon>0$, there exists a finitely enumerable $\varepsilon$-approximation to $\Delta$ (a set $S$ is finitely enumerable if there exist a natural number $N$ and a mapping of the set $\{1,2, \ldots, N\}$ onto $S)$. An $\varepsilon$-approximation to $\Delta$ is a subset of $\Delta$ such that for each $\mathbf{p} \in \Delta$, there exists $\mathbf{q}$ in that $\varepsilon$-approximation with $|\mathbf{p}-\mathbf{q}|<\varepsilon$. Each face (boundary) 
of $\Delta$ is also a simplex, and so it is compact. According to Corollary 2.2.12 of [9], we have the following result.

Lemma 3. For each $\varepsilon>0$, there exist totally bounded sets $H_{1}$, $H_{2}, \ldots, H_{n}$, each of diameter less than or equal to $\varepsilon$, such that $\Delta=\bigcup_{i=1}^{n} H_{i}$. lows.

The definition of sequential local nonconstancy is as fol-

Definition 4 (sequential local nonconstancy of functions). There exists $\bar{\varepsilon}$ with the following property. For each $\varepsilon>0$ less than $\bar{\varepsilon}$, there exist totally bounded sets $H_{1}, H_{2}, \ldots, H_{m}$, each of diameter less than or equal to $\varepsilon$, such that $\Delta=\bigcup_{i=1}^{m} H_{i}$, and if for all sequences $\left(\mathbf{p}_{n}\right)_{n \geq 1},\left(\mathbf{q}_{n}\right)_{n \geq 1}$ in each $H_{i},\left|\varphi\left(\mathbf{p}_{n}\right)-\mathbf{p}_{n}\right| \rightarrow$ 0 and $\left|\varphi\left(\mathbf{q}_{n}\right)-\mathbf{q}_{n}\right| \rightarrow 0$, then $\left|\mathbf{p}_{n}-\mathbf{q}_{n}\right| \rightarrow 0$.

We show the following lemma.

Lemma 5. Let $\varphi$ be a uniformly continuous and sequentially locally nonconstant function from $\Delta$ to itself. Assume that $\inf _{\mathbf{p} \in H_{i}} \varphi(\mathbf{p})=0$ for $H_{i} \subset \Delta$ defined above. If the following property holds: for each $\varepsilon>0$, there exists $\delta>0$ such that if $\mathbf{p}, \mathbf{q} \in H_{i},|\varphi(\mathbf{p})-\mathbf{p}|<\delta$, and $|\varphi(\mathbf{q})-\mathbf{q}|<\delta$, then $|\mathbf{p}-\mathbf{q}| \leq \varepsilon$, then there exists a point $\mathbf{r} \in H_{i}$ such that $\varphi(\mathbf{r})=\mathbf{r}$.

Proof. Choose a sequence $\left(\mathbf{p}_{n}\right)_{n \geq 1}$ in $H_{i}$ such that $\mid \varphi\left(\mathbf{p}_{n}\right)-$ $\mathbf{p}_{n} \mid \rightarrow 0$. Compute $N$ such that $\left|\varphi\left(\mathbf{p}_{n}\right)-\mathbf{p}_{n}\right|<\delta$ for all $n \geq N$. Then, for $m, n \geq N$, we have $\left|\mathbf{p}_{m}-\mathbf{p}_{n}\right| \leq \varepsilon$. Since $\varepsilon>0$ is arbitrary, $\left(\mathbf{p}_{n}\right)_{n \geq 1}$ is a Cauchy sequence in $H_{i}$ and converges to a limit $\mathbf{r} \in H_{i}$. The continuity of $\varphi$ yields $|\varphi(\mathbf{r})-\mathbf{r}|=0$, that is, $\varphi(\mathbf{r})=\mathbf{r}$.

Now, we look at the problem of the existence of a Nash equilibrium in a finite strategic game according to [13]. A Nash equilibrium of a finite strategic game is a state where all players choose their best responses to strategies of other players.

Consider an $n$-players strategic game with $m$ pure strategies for each player. $n$ and $m$ are finite natural numbers not smaller than 2. Let $S_{i}$ be the set of pure strategies of player $i$, and denote each of his pure strategies by $s_{i j}$. His mixed strategy is defined as a probability distribution over $S_{i}$ and is denoted by $\mathbf{p}_{i}$. Let $p_{i j}$ be a probability that player $i$ chooses $s_{i j}$, then we must have $\sum_{j=1}^{m} p_{i j}=1$ for all $i$. A combination of mixed strategies of all players is called a profile. It is denoted by $\mathbf{p}$. Let $\pi_{i}(\mathbf{p})$ be the expected payoff of player $i$ at profile $\mathbf{p}$, and let $\pi_{i}\left(s_{i j}, \mathbf{p}_{-i}\right)$ be his payoff when he chooses a strategy $s_{i j}$ at that profile, where $\mathbf{p}_{-i}$ denotes a combination of mixed strategies of players other than $i$ at profile $\mathbf{p} . \pi_{i}(\mathbf{p})$ is written as follows:

$$
\pi_{i}(\mathbf{p})=\pi_{i}\left(\mathbf{p}_{i}, \mathbf{p}_{-i}\right)=\sum_{\left\{j: p_{i j}>0\right\}} p_{i j} \pi_{i}\left(s_{i j}, \mathbf{p}_{-i}\right) .
$$

Assume that the values of payoffs of all players are finite, then since pure strategies are finite, and expected payoffs are linear functions about probability distributions over the sets of pure strategies of all players, $\pi_{i}(\mathbf{p})$ is uniformly continuous about $\mathbf{p}$.
For each $i$ and $j$, let

$$
v_{i j}=p_{i j}+\max \left(\pi_{i}\left(s_{i j}, \mathbf{p}_{-i}\right)-\pi_{i}(\mathbf{p}), 0\right),
$$

and define the following function:

$$
\psi_{i j}(\mathbf{p})=\frac{v_{i j}}{v_{i 1}+v_{i 2}+\cdots+v_{i m}},
$$

where $\sum_{j=1}^{m} \psi_{i j}=1$ for all $i$. Let $\psi_{i}(\mathbf{p})=\left(\psi_{i 1}, \psi_{i 2}, \ldots, \psi_{i m}\right)$, $\psi(\mathbf{p})=\left(\psi_{1}, \psi_{2}, \ldots, \psi_{n}\right)$. Since each $\psi_{i}$ is an $m$-dimensional vector such that the values of its components are between 0 and 1 , and the sum of its components is 1 , it represents a point on an $m$-1-dimensional simplex. $\psi(\mathbf{p})$ is a combination of vectors $\psi_{i}$ 's. It is a vector, such that its components are components of $\psi_{i}(\mathbf{p})$ for all players. Thus, it is a vector with $n \times m$ components, but since the number of independent components is $n(m-1)$, the range of $\psi$ is the $n$-times product of $m-1$-dimensional simplices. It is convex, and homeomorphic to an $n(m-1)$-dimensional simplex. $\mathbf{p}=\left(\mathbf{p}_{1}, \mathbf{p}_{2}, \ldots, \mathbf{p}_{n}\right)$ is also a vector with $n \times m$ components, and the number of its independent components is $n(m-1)$.

Let us consider a homeomorphism between an $n(m-$ 1)-dimensional simplex and the space of players' mixed strategies which is denoted by $\mathbf{P}$. Figure 2 depicts an example of a case of two players with two pure strategies for each player. Vertices $D, E, F$, and $G$ represent states where two players choose pure strategies, and points on edges $D E, E F$, $F G$, and $G D$ represent states where one player chooses a pure strategy. Vertices of the simplex and points on faces (simplices whose dimension is lower than $n(m-1)$ ) of the simplex correspond to the points on faces of $\mathbf{P}$. For example, in Figure 2, A, B and C correspond, respectively, to $I, J$, and $H$. On the other hand, each vertex of $\mathbf{P}, D, E, F$, and $G$ corresponds, respectively, to itself on a face of the simplex which contains it.

Next, we assume the following condition.

Definition 6 (sequential local nonconstancy of payoff functions). There exists $\bar{\varepsilon}$ with the following property. For each $\varepsilon>0$ less than $\bar{\varepsilon}$, there exist totally bounded sets $H_{1}$, $H_{2}, \ldots, H_{m}$, each of diameter less than or equal to $\varepsilon$, such that $\mathbf{P}=\bigcup_{i=1}^{m} H_{i}$, and if for all sequences $\left(\mathbf{p}_{n}\right)_{n \geq 1},\left(\mathbf{q}_{n}\right)_{n \geq 1}$ in each $H_{i}, \max \left(\pi_{i}\left(s_{i j},\left(\mathbf{p}_{n}\right)_{-i}\right)-\pi_{i}\left(\mathbf{p}_{n}\right), 0\right) \rightarrow 0, \max \left(\pi_{i}\left(s_{i j},\left(\mathbf{q}_{n}\right)_{-i}\right)-\right.$ $\left.\pi_{i}\left(\mathbf{q}_{n}\right), 0\right) \rightarrow 0$ for all $s_{i j} \in S_{i}$ for all $i$, then $\left|\mathbf{p}_{n}-\mathbf{q}_{n}\right| \rightarrow 0$.

By the sequential local nonconstancy of payoff functions we obtain the following result.

For each $\varepsilon>0$ less than $\bar{\varepsilon}$, there exist totally bounded sets $H_{1}, H_{2}, \ldots, H_{m}$, each of diameter less than or equal to $\varepsilon$, such that $\mathbf{P}=\bigcup_{i=1}^{m} H_{i}$, and if for all sequences $\left(\mathbf{p}_{n}\right)_{n \geq 1},\left(\mathbf{q}_{n}\right)_{n \geq 1}$ in each $H_{i},\left|\psi\left(\mathbf{p}_{n}\right)-\mathbf{p}_{n}\right| \rightarrow 0$ and $\left|\psi\left(\mathbf{q}_{n}\right)-\mathbf{q}_{n}\right| \rightarrow 0$, then $\left|\mathbf{p}_{n}-\mathbf{q}_{n}\right| \rightarrow 0$.

Thus, $\psi$ is sequentially locally nonconstant.

Let us replace $n$ by $n(m-1)$. We show the following theorem.

Theorem 7. In any finite strategic game with sequentially locally nonconstant payoff functions, there exists a Nash equilibrium. 


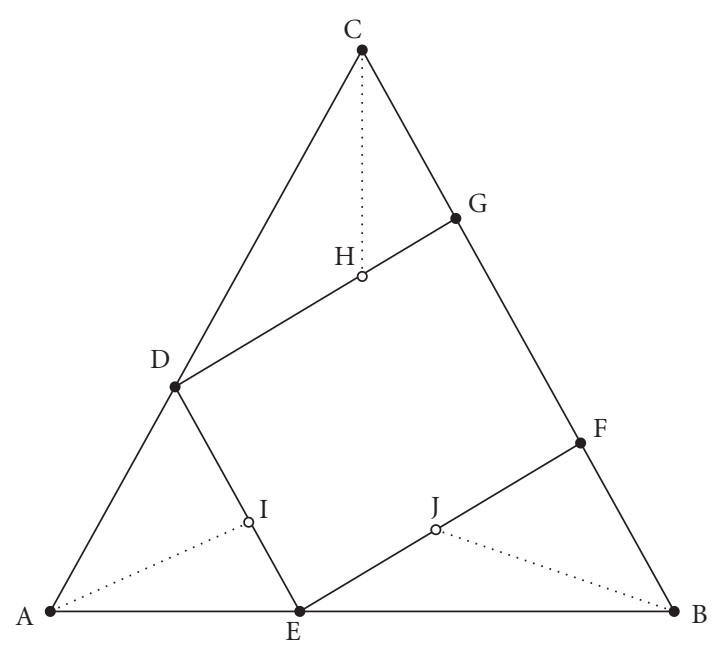

FIGURE 2: Homeomorphism between simplex and combination of strategies.

Proof. Let us prove this theorem through some steps.

(1) First we show that we can partition an $n(m-1)$ dimensional simplex $\Delta$, so that the conditions for Sperner's lemma are satisfied. We partition $\Delta$ according to the method in the proof of Sperner's lemma and label the vertices of simplices constructed by partition of $\Delta$. It is important how to label the vertices contained in the faces of $\Delta$. Let $K$ be the set of small simplices constructed by partition of $\Delta$, let $\mathbf{p}=\left(\mathbf{p}_{0}, \mathbf{p}_{1}, \ldots, \mathbf{p}_{n(m-1)}\right)$ be a vertex of a simplex of $K$, and denote the $i$ th coordinate of $\psi(\mathbf{p})$ by $\psi_{i}$ or $\psi_{i}(\mathbf{p})$. We label a vertex $\mathbf{p}$ according to the following rule:

$$
\text { if } \mathbf{p}_{k}+\tau>\psi_{k} \text {, we label } \mathbf{p} \text { with } k \text {. }
$$

$\tau$ is an arbitrary positive number. If there are multiple $k$ 's which satisfy this condition, we label $\mathbf{p}$ conveniently for the conditions for Sperner's lemma to be satisfied.

For example, let $\mathbf{p}$ be a point contained in an $n(m-1)-$ 1-dimensional face of $\Delta$ such that $p_{i}=0$ for one $i$ among $0,1,2, \ldots, n(m-1)$. With $\tau>0$, we have $f_{i}>0$ or $f_{i}<\tau$ (in constructive mathematics for any real number $x$ we can not prove that $x \geq 0$ or $x<0$, that, $x>0, x=0$, or $x<0$. But for any distinct real numbers $x, y$, and $z$ such that $x>z$, we can prove that $x>y$ or $y>z)$. When $\psi_{i}>0$, from $\sum_{j=0}^{n(m-1)} \mathbf{p}_{j}=1$, $\sum_{j=0}^{n(m-1)} \psi_{j}=1$, and $\mathbf{p}_{i}=0$,

$$
\sum_{j=0, j \neq i}^{n(m-1)} \mathbf{p}_{j}>\sum_{j=0, j \neq i}^{n(m-1)} \psi_{j} .
$$

Then, for at least one $j$ (denote it by $k$ ), we have $\mathbf{p}_{k}>\psi_{k}$, and we label $\mathbf{p}$ with $k$, where $k$ is one of the numbers which satisfy $\mathbf{p}_{k}>\psi_{k}$. Since $\psi_{i}>\mathbf{p}_{i}=0, i$ does not satisfy this condition. Assume that $\psi_{i}<\tau \cdot \mathbf{p}_{i}=0$ implies $\sum_{j=0, j \neq i}^{n(m-1)} \mathbf{p}_{j}=1$. Since $\sum_{j=0, j \neq i}^{n(m-1)} \psi_{j} \leq 1$, we obtain

$$
\sum_{j=0, j \neq i}^{n(m-1)} \mathbf{p}_{j} \geq \sum_{j=0, j \neq i}^{n(m-1)} \psi_{j}
$$

Then, for a positive number $\tau$, we have

$$
\sum_{j=0, j \neq i}^{n(m-1)}\left(\mathbf{p}_{j}+\tau\right)>\sum_{j=0, j \neq i}^{n(m-1)} \psi_{j} .
$$

There is at least one $j(\neq i)$ which satisfies $\mathbf{p}_{j}+\tau>\psi_{j}$. Denote it by $k$, and we label $\mathbf{p}$ with $k$. $k$ is one of the numbers other than $i$ such that $\mathbf{p}_{k}+\tau>\psi_{k}$ is satisfied. $i$ itself satisfies this condition $\left(\mathbf{p}_{i}+\tau>\psi_{i}\right)$. But, since there is a number other than $i$ which satisfies this condition, we can select a number other than $i$. We have proved that we can label the vertices contained in an $n(m-1)-1$-dimensional face of $\Delta$ such that $\mathbf{p}_{i}=0$ for one $i$ among $0,1,2, \ldots, n(m-1)$ with the numbers other than $i$. By similar procedures, we can show that we can label the vertices contained in an $n(m-1)-2$ dimensional face of $\Delta$ such that $\mathbf{p}_{i}=0$ for two $i$ s among $0,1,2, \ldots, n(m-1)$ with the numbers other than those $i$ 's, and so on.

Consider the case where $\mathbf{p}_{i}=\mathbf{p}_{i+1}=0$. We see that when $\psi_{i}>0$ or $\psi_{i+1}>0$,

$$
\sum_{j=0, j \neq i, i+1}^{n(m-1)} \mathbf{p}_{j}>\sum_{j=0, j \neq i, i+1}^{n(m-1)} \psi_{j},
$$

and so for at least one $j$ (denote it by $k$ ), we have $\mathbf{p}_{k}>\psi_{k}$, and we label $\mathbf{p}$ with $k$. On the other hand, when $\psi_{i}<\tau$ and $\psi_{i+1}<\tau$, we have

$$
\sum_{j=0, j \neq i, i+1}^{n(m-1)} \mathbf{p}_{j} \geq \sum_{j=0, j \neq i, i+1}^{n(m-1)} \psi_{j} .
$$

Then, for a positive number $\tau$, we have

$$
\sum_{j=0, j \neq i, i+1}^{n(m-1)}\left(\mathbf{p}_{j}+\tau\right)>\sum_{j=0, j \neq i, i+1}^{n(m-1)} \psi_{j} .
$$

Thus, there is at least one $j(\neq i, i+1)$ which satisfies $\mathbf{p}_{j}+\tau>$ $\psi_{j}$. Denote it by $k$, and we label $\mathbf{p}$ with $k$. 
Next, consider the case where $\mathbf{p}_{i}=0$ for all $i$ other than $n(m-1)$. If for some $i \psi_{i}>0$, then we have $\mathbf{p}_{n(m-1)}>\psi_{n(m-1)}$ and label $\mathbf{p}$ with $n(m-1)$. On the other hand, if $\psi_{j}<\tau$ for all $j \neq n(m-1)$, then we obtain $\mathbf{p}_{n(m-1)} \geq \psi_{n(m-1)}$. It implies $\mathbf{p}_{n(m-1)}+\tau>\psi_{n(m-1)}$. Thus, we can label $\mathbf{p}$ with $n(m-1)$.

Therefore, the conditions for Sperner's lemma are satisfied, and there exists an odd number of fully labeled simplices in $K$.

(2) Suppose that we partition $\Delta$ sufficiently fine so that the distance between any pair of the vertices of simplices of $K$ is sufficiently small. Let $\delta^{n(m-1)}$ be a fully labeled $n(m-1)$-dimensional simplex of $K$, and let $\mathbf{p}^{0}, \mathbf{p}^{1}, \ldots$ and $\mathbf{p}^{n(m-1)}$ be the vertices of $\delta^{n(m-1)}$. We name these vertices so that $\mathbf{p}^{0}, \mathbf{p}^{1}, \ldots, \mathbf{p}^{n(m-1)}$ are labeled, respectively, with $0,1, \ldots$, $n(m-1)$. The values of $\psi$ at these vertices are $\psi\left(\mathbf{p}^{0}\right), \psi\left(\mathbf{p}^{1}\right), \ldots$ and $\psi\left(\mathbf{p}^{n(m-1)}\right)$. The $j$ th coordinates of $\mathbf{p}^{i}$ and $\psi\left(\mathbf{p}^{i}\right), i=$ $0,1, \ldots, n(m-1)$, are, respectively, denoted by $\mathbf{p}_{j}^{i}$ and $\psi_{j}\left(\mathbf{p}^{i}\right)$. About $\mathbf{p}^{0}$, from the labeling rules, we have $\mathbf{p}_{0}^{0}+\tau>\psi_{0}\left(\mathbf{p}^{0}\right)$. About $\mathbf{p}^{1}$, also from the labeling rules, we have $\mathbf{p}_{1}^{1}+\tau>$ $\psi_{1}\left(\mathbf{p}^{1}\right)$. Since $n$ and $m$ are finite, by the uniform continuity of $\psi$ there exists $\delta>0$ such that if $\left|\mathbf{p}^{i}-\mathbf{p}^{j}\right|<\delta$, then $\left|\psi\left(\mathbf{p}^{i}\right)-\psi\left(\mathbf{p}^{j}\right)\right|<\varepsilon / 2 n(m-1)[n(m-1)+1]$ for $\varepsilon>0$ and $i \neq j .\left|\psi\left(\mathbf{p}^{0}\right)-\psi\left(\mathbf{p}^{1}\right)\right|<\varepsilon / 2 n(m-1)[n(m-1)+1]$ means $\psi_{1}\left(\mathbf{p}^{1}\right)>\psi_{1}\left(\mathbf{p}^{0}\right)-\varepsilon / 2 n(m-1)[n(m-1)+1]$. On the other hand, $\left|\mathbf{p}^{0}-\mathbf{p}^{1}\right|<\delta$ means that $\mathbf{p}_{1}^{0}>\mathbf{p}_{1}^{1}-\delta$. We can make $\delta$ satisfy $\delta<\varepsilon / 2 n(m-1)[n(m-1)+1]$. Thus, from

$$
\begin{gathered}
\mathbf{p}_{1}^{0}>\mathbf{p}_{1}^{1}-\delta, \quad \mathbf{p}_{1}^{1}+\tau>\psi_{1}\left(\mathbf{p}^{1}\right), \\
\psi_{1}\left(\mathbf{p}^{1}\right)>\psi_{1}\left(\mathbf{p}^{0}\right)-\frac{\varepsilon}{2 n(m-1)[n(m-1)+1]},
\end{gathered}
$$

we obtain

$$
\begin{aligned}
\mathbf{p}_{1}^{0} & >\psi_{1}\left(\mathbf{p}^{0}\right)-\delta-\tau-\frac{\varepsilon}{2 n(m-1)[n(m-1)+1]} \\
& >\psi_{1}\left(\mathbf{p}^{0}\right)-\frac{\varepsilon}{n(m-1)[n(m-1)+1]}-\tau .
\end{aligned}
$$

By similar arguments, for each $i$ other than 0 ,

$$
\mathbf{p}_{i}^{0}>\psi_{i}\left(\mathbf{p}^{0}\right)-\frac{\varepsilon}{n(m-1)[n(m-1)+1]}-\tau .
$$

For $i=0$, we have

$$
\mathbf{p}_{0}^{0}>\psi_{0}\left(\mathbf{p}^{0}\right)-\tau \text {. }
$$

Adding (13) and (14) side by side except for some $i$ (denote it by $k$ ) other than 0 ,

$$
\begin{aligned}
\sum_{j=0, j \neq k}^{n(m-1)} \mathbf{p}_{j}^{0}> & \sum_{j=0, j \neq k}^{n(m-1)} \psi_{j}\left(\mathbf{p}^{0}\right) \\
& -\frac{[n(m-1)-1] \varepsilon}{n(m-1)[n(m-1)+1]}-n(m-1) \tau .
\end{aligned}
$$

From $\sum_{j=0}^{n(m-1)} \mathbf{p}_{j}^{0}=1, \sum_{j=0}^{n(m-1)} \psi_{j}\left(\mathbf{p}^{0}\right)=1$, we have $1-\mathbf{p}_{k}^{0}>$ $1-\psi_{k}\left(\mathbf{p}^{0}\right)-([n(m-1)-1] \varepsilon /(n(m-1)[n(m-1)+1]))-$ $n(m-1) \tau$, which is rewritten as

$$
\mathbf{p}_{k}^{0}<\psi_{k}\left(\mathbf{p}^{0}\right)+\frac{[n(m-1)-1] \varepsilon}{n(m-1)[n(m-1)+1]}+n(m-1) \tau .
$$

Since (13) implies $\mathbf{p}_{k}^{0}>\psi_{k}\left(\mathbf{p}^{0}\right)-\varepsilon /(n(m-1)[n(m-1)+1])-\tau$, we have

$$
\begin{aligned}
\psi_{k}\left(\mathbf{p}^{0}\right) & -\frac{\varepsilon}{n(m-1)[n(m-1)+1]}-\tau \\
& <\mathbf{p}_{k}^{0}<\psi_{k}\left(\mathbf{p}^{0}\right)+\frac{[n(m-1)-1] \varepsilon}{n(m-1)[n(m-1)+1]}+n(m-1) \tau .
\end{aligned}
$$

Thus,

$$
\left|\mathbf{p}_{k}^{0}-\psi_{k}\left(\mathbf{p}^{0}\right)\right|<\frac{[n(m-1)-1] \varepsilon}{n(m-1)[n(m-1)+1]}+n(m-1) \tau
$$

is derived. On the other hand, adding (13) from 1 to $n(m-1)$ yields

$$
\sum_{j=1}^{n(m-1)} \mathbf{p}_{j}^{0}>\sum_{j=1}^{n(m-1)} \psi_{j}\left(\mathbf{p}^{0}\right)-\frac{\varepsilon}{n(m-1)+1}-n(m-1) \tau .
$$

From $\sum_{j=0}^{n(m-1)} \mathbf{p}_{j}^{0}=1, \sum_{j=0}^{n(m-1)} \psi_{j}\left(\mathbf{p}^{0}\right)=1$, we have

$$
1-\mathbf{p}_{0}^{0}>1-\psi_{0}\left(\mathbf{p}^{0}\right)-\frac{\varepsilon}{n(m-1)+1}-n(m-1) \tau .
$$

Then, from (14) and (20), we get

$$
\left|\mathbf{p}_{0}^{0}-\psi_{0}\left(\mathbf{p}^{0}\right)\right|<\frac{\varepsilon}{n(m-1)+1}+n(m-1) \tau .
$$

From (18) and (21), we obtain the following result:

$$
\left|\mathbf{p}_{i}^{0}-\psi_{i}\left(\mathbf{p}^{0}\right)\right|<\frac{\varepsilon}{n(m-1)+1}+n(m-1) \tau \quad \forall i .
$$

Thus,

$$
\left|\mathbf{p}^{0}-\psi\left(\mathbf{p}^{0}\right)\right|<\varepsilon+n(m-1)[n(m-1)+1] \tau .
$$

Since $\varepsilon$ and $\tau$ are arbitrary, we have $\inf _{\mathbf{p} \in \Delta}|\psi(\mathbf{p})-\mathbf{p}|=0$.

(3) Since, by Lemma 3, $\Delta=\sum_{i=1}^{n} H_{i}$, where each $H_{i}$ is a totally bounded set whose diameter is less than or equal to $\varepsilon$, we have $\inf _{\mathbf{p} \in H_{i}}|\psi(\mathbf{p})-\mathbf{p}|=0$ for at least one $H_{i}$. Choose a sequence $\left(\mathbf{r}_{n}\right)_{n \geq 1}$ such that $\left|\psi\left(\mathbf{r}_{n}\right)-\mathbf{r}_{n}\right| \rightarrow 0$ in such $H_{i}$. In view of Lemma 5, it is enough to prove that the following condition holds.

For each $\varepsilon>0$, there exists $\delta>0$ such that if $\mathbf{p}, \mathbf{q} \in H_{i}$, $|\psi(\mathbf{p})-\mathbf{p}|<\delta$, and $|\psi(\mathbf{q})-\mathbf{q}|<\delta$, then $|\mathbf{p}-\mathbf{q}| \leq \varepsilon$.

Assume that the set

$$
T=\left\{(\mathbf{p}, \mathbf{q}) \in H_{i} \times H_{i}:|\mathbf{p}-\mathbf{q}| \geq \varepsilon\right\}
$$

is nonempty and compact (see Theorem 2.2.13 of [9]). Since the mapping $(\mathbf{p}, \mathbf{q}) \rightarrow \max (|\psi(\mathbf{p})-\mathbf{p}|,|\psi(\mathbf{q})-\mathbf{q}|)$ is uniformly continuous, we can construct an increasing binary sequence $\left(\lambda_{n}\right)_{n \geq 1}$ such that

$$
\begin{aligned}
& \lambda_{n}=0 \Longrightarrow \inf _{(\mathbf{p}, \mathbf{q}) \in T} \max (|\psi(\mathbf{p})-\mathbf{p}|,|\psi(\mathbf{q})-\mathbf{q}|)<2^{-n}, \\
& \lambda_{n}=1 \Longrightarrow \inf _{(\mathbf{p}, \mathbf{q}) \in T} \max (|\psi(\mathbf{p})-\mathbf{p}|,|\psi(\mathbf{q})-\mathbf{q}|)>2^{-n-1} .
\end{aligned}
$$


TABLE 1: Example of game 1.

\begin{tabular}{llcc}
\hline & & & Player 2 \\
\hline \multirow{2}{*}{ Player 1 } & & $\mathrm{X}$ & $\mathrm{Y}$ \\
& $\mathrm{X}$ & 2,2 & 0,3 \\
& $\mathrm{Y}$ & 3,0 & 1,1 \\
\hline
\end{tabular}

It suffices to find $n$ such that $\lambda_{n}=1$. In that case, if $\mid \psi(\mathbf{p})-$ $\mathbf{p}\left|<2^{-n-1},\right| \psi(\mathbf{q})-\mathbf{q} \mid<2^{-n-1}$, we have $(\mathbf{p}, \mathbf{q}) \notin T$ and $|\mathbf{p}-\mathbf{q}| \leq \varepsilon$. Assume that $\lambda_{1}=0$. If $\lambda_{n}=0$, choose $\left(\mathbf{p}_{n}, \mathbf{q}_{n}\right) \in$ $T$ such that $\max \left(\left|\psi\left(\mathbf{p}_{n}\right)-\mathbf{p}_{n}\right|,\left|\psi\left(\mathbf{q}_{n}\right)-\mathbf{q}_{n}\right|\right)<2^{-n}$, and if $\lambda_{n}=$ 1 , set $\mathbf{p}_{n}=\mathbf{q}_{n}=\mathbf{r}_{n}$. Then, $\left|\psi\left(\mathbf{p}_{n}\right)-\mathbf{p}_{n}\right| \rightarrow 0$ and $\mid \psi\left(\mathbf{q}_{n}\right)-$ $\mathbf{q}_{n} \mid \rightarrow 0$, so $\left|\mathbf{p}_{n}-\mathbf{q}_{n}\right| \rightarrow 0$. Computing $N$ such that $\mid \mathbf{p}_{N}-$ $\mathbf{q}_{N} \mid<\varepsilon$, we must have $\lambda_{N}=1$. We have completed the proof of the existence of a point which satisfies $\psi(\mathbf{p})=\mathbf{p}$.

(4) Denote one of the points which satisfy $\psi(\mathbf{p})=\mathbf{p}$ by $\widetilde{\mathbf{p}}=\left(\widetilde{p}_{1}, \tilde{p}_{2}, \ldots, \widetilde{p}_{n}\right)$ and the components of $\widetilde{p}_{i}$ by $\widetilde{p}_{i j}$. Then, we have

$$
\psi_{i j}=\tilde{p}_{i j}, \quad \forall i, j
$$

By the definition of $\psi_{i j}$,

$$
\frac{\tilde{p}_{i j}+\max \left(\pi_{i}\left(s_{i j}, \widetilde{\mathbf{p}}_{-i}\right)-\pi_{i}(\widetilde{\mathbf{p}}), 0\right)}{1+\sum_{k=1}^{m} \max \left(\pi_{i}\left(s_{i k}, \widetilde{\mathbf{p}}_{-i}\right)-\pi_{i}(\widetilde{\mathbf{p}}), 0\right)}=\tilde{p}_{i j} .
$$

Let $\lambda=\sum_{k=1}^{m} \max \left(\pi_{i}\left(s_{i k}, \widetilde{\mathbf{p}}_{-i}\right)-\pi_{i}(\widetilde{\mathbf{p}}), 0\right)$, then

$$
\max \left(\pi_{i}\left(s_{i j}, \widetilde{\mathbf{p}}_{-i}\right)-\pi_{i}(\tilde{\mathbf{p}}), 0\right)=\lambda \tilde{p}_{i j}
$$

where $\widetilde{\mathbf{p}}_{-i}$ denotes a combination of mixed strategies of players other than $i$ at profile $\tilde{\mathbf{p}}$.

Since $\pi_{i}(\tilde{\mathbf{p}})=\sum_{\left\{j: \tilde{p}_{i j}>0\right\}} \tilde{p}_{i j} \pi_{i}\left(s_{i j}, \widetilde{\mathbf{p}}_{i}\right)$, it is impossible that $\max \left(\pi_{i}\left(s_{i j}, \widetilde{\mathbf{p}}_{-i}\right)-\pi_{i}(\widetilde{\mathbf{p}}), 0\right)=\pi_{i}\left(s_{i j}, \widetilde{\mathbf{p}}_{-i}\right)-\pi_{i}(\widetilde{\mathbf{p}})>0$ for all $j$ satisfying $\tilde{p}_{i j}>0$. Thus, $\lambda=0$, and $\max \left(\pi_{i}\left(s_{i j}, \widetilde{\mathbf{p}}_{-i}\right)-\right.$ $\left.\pi_{i}(\tilde{\mathbf{p}}), 0\right)=0$ holds for all $s_{i j}$ 's whether $\tilde{p}_{i j}>0$ or not, and it holds for all players. Then, strategies of all players in $\tilde{\mathbf{p}}$ are the best responses to each other, and a state where all players choose these strategies is a Nash equilibrium.

Consider two examples. See a game in Table 1. It is an example of the so-called Prisoners' Dilemma. Pure strategies of players 1 and 2 are $X$ and $Y$. The left-side number in each cell represents the payoff of player 1 , and the right-side number represents the payoff of player 2 . Let $p_{X}$ and $1-p_{X}$ denote the probabilities that player 1 chooses, respectively, $X$ and $Y$, and $q_{X}$ and $1-q_{X}$ denote the probabilities for player 2 . Denote the expected payoffs of players 1 and 2 by $\pi_{1}\left(p_{X}, q_{X}\right)$ and $\pi_{2}\left(p_{X}, q_{X}\right)$, then

$$
\begin{aligned}
\pi_{1}\left(p_{X}, q_{X}\right) & =2 p_{X} q_{X}+3\left(1-p_{X}\right) q_{X}+\left(1-p_{X}\right)\left(1-q_{X}\right) \\
& =1-p_{X}+2 q_{X} \\
\pi_{2}\left(p_{X}, q_{X}\right) & =2 p_{X} q_{X}+3 p_{X}\left(1-q_{X}\right)+\left(1-p_{X}\right)\left(1-q_{X}\right) \\
& =1-q_{X}+2 p_{X} .
\end{aligned}
$$

TABLE 2: Example of game 2.

\begin{tabular}{lccc}
\hline & & & Player 2 \\
\hline \multirow{2}{*}{ Player 1 } & & $\mathrm{X}$ & $\mathrm{Y}$ \\
& $\mathrm{X}$ & 2,1 & 0,0 \\
& $\mathrm{Y}$ & 0,0 & 1,2 \\
\hline
\end{tabular}

Denote the payoff of player 1 when he chooses $X$ by $\pi_{1}\left(X, q_{X}\right)$ and that when he chooses $Y$ by $\pi_{1}\left(Y, q_{X}\right)$. Do similarly for Player B, then

$$
\begin{aligned}
& \pi_{1}\left(Y, q_{X}\right)=1+2 q_{X}>\pi_{1}\left(p_{X}, q_{X}\right) \text { for any } q_{X}, p_{X}>0 \\
& \pi_{2}\left(p_{X}, Y\right)=1+2 p_{X}>\pi_{2}\left(p_{X}, q_{X}\right) \text { for any } p_{X}, q_{X}>0 .
\end{aligned}
$$

Consider two sequences of $p_{X}, \quad\left(p_{X}(m)\right)_{m \geq 1}$ and $\left(p_{X}^{\prime}(m)\right)_{m \geq 1}$, such that $p_{X}(m)>0$ and $p_{X}^{\prime}(m)>0$. If $\max \left(\max \left(\pi_{1}\left(X, q_{X}\right), \pi_{1}\left(Y, q_{X}\right)\right)-\pi_{1}\left(p_{X}(m), q_{X}\right), 0\right)=$ $\max \left(\pi_{1}\left(Y, q_{X}\right)-\pi_{1}\left(p_{X}(m), q_{X}\right), 0\right) \rightarrow 0$ and $\max \left(\max \left(\pi_{1}(X\right.\right.$, $\left.\left.\left.q_{X}\right), \pi_{1}\left(Y, q_{X}\right)\right)-\pi_{1}\left(p_{X}^{\prime}(m), q_{X}\right), 0\right)=\max \left(\pi_{1}\left(Y, q_{X}\right)-\right.$ $\left.\pi_{1}\left(p_{X}^{\prime}(m), q_{X}\right), 0\right) \rightarrow 0$, then $p_{X}(m) \rightarrow 0, p_{X}^{\prime}(m) \rightarrow 0$, and $\left|p_{X}(m)-p_{X}^{\prime}(m)\right| \rightarrow 0$.

Consider two sequences of $q_{X},\left(q_{X}(m)\right)_{m \geq 1}$ and $\left(q_{X}^{\prime}(m)\right)_{m \geq 1}$, such that $q_{X}(m)>0$ and $q_{X}^{\prime}(m)>0$. If $\max \left(\max \left(\pi_{2}\left(p_{X}, X\right), \pi_{2}\left(p_{X}, Y\right)\right)-\pi_{2}\left(p_{X}, q_{X}(m)\right), 0\right)=$ $\max \left(\pi_{2}\left(p_{X}, Y\right)-\pi_{2}\left(p_{X}, q_{X}(m)\right), 0\right) \rightarrow 0$ and $\max \left(\max \left(\pi_{2}\left(p_{X}\right.\right.\right.$, $\left.\left.X), \pi_{2}\left(p_{X}, Y\right)\right)-\pi_{2}\left(p_{X}, q_{X}^{\prime}(m)\right), 0\right)=\max \left(\pi_{2}\left(p_{X}, Y\right)-\right.$ $\left.\pi_{2}\left(p_{X}, q_{X}^{\prime}(m)\right), 0\right) \rightarrow 0$, then $q_{X}(m) \rightarrow 0, q_{X}^{\prime}(m) \rightarrow 0$, and $\left|q_{X}(m)-q_{X}^{\prime}(m)\right| \rightarrow 0$.

Therefore, the payoff functions are sequentially locally nonconstant.

Let us consider another example. See a game in Table 2. It is an example of the so-called Battle of the Sexes Game. Notations are the same as those in the previous example. The expected payoffs of players are as follows:

$$
\begin{aligned}
\pi_{1}\left(p_{X}, q_{X}\right) & =2 p_{X} q_{X}+\left(1-p_{X}\right)\left(1-q_{X}\right) \\
& =1+p_{X}\left(3 q_{X}-1\right)-q_{X}, \\
\pi_{1}\left(X, q_{X}\right) & =2 q_{X}, \\
\pi_{1}\left(Y, q_{X}\right) & =1-q_{X}, \\
\pi_{2}\left(p_{X}, q_{X}\right) & =p_{X} q_{X}+2\left(1-p_{X}\right)\left(1-q_{X}\right) \\
& =2+q_{X}\left(3 p_{X}-2\right)-2 p_{X}, \\
\pi_{2}\left(p_{X}, X\right) & =p_{X}, \\
\pi_{2}\left(p_{X}, Y\right) & =2-2 p_{X} .
\end{aligned}
$$

Then,

(i) when $q_{X}>1 / 3, \pi_{1}\left(X, q_{X}\right)>\pi_{1}\left(p_{X}, q_{X}\right)$ for $p_{X}<1$,

(ii) when $q_{X}<1 / 3, \pi_{1}\left(Y, q_{X}\right)>\pi_{1}\left(p_{X}, q_{X}\right)$ for $p_{X}>0$,

(iii) when $p_{X}>2 / 3, \pi_{2}\left(p_{X}, X\right)>\pi_{2}\left(p_{X}, q_{X}\right)$ for $q_{X}<1$,

(iv) when $p_{X}<2 / 3, \pi_{2}\left(p_{X}, Y\right)>\pi_{2}\left(p_{X}, q_{X}\right)$ for $q_{X}>0$.

Consider sequences $\left(p_{X}(m)\right)_{m \geq 1},\left(p_{X}^{\prime}(m)\right)_{m \geq 1},\left(q_{X}(m)\right)_{m \geq 1}$, and $\left(q_{X}^{\prime}(m)\right)_{m \geq 1}$. 
(1) When $p_{X}>2 / 3, q_{X}>1 / 3$, if $\max \left(\pi_{1}\left(X, q_{X}\right)-\right.$ $\left.\pi_{1}\left(p_{X}(m), q_{X}\right), 0\right) \rightarrow 0$ and $\max \left(\pi_{1}\left(X, q_{X}\right)-\pi_{1}\left(p_{X}^{\prime}(m)\right.\right.$, $\left.\left.q_{X}\right), 0\right) \rightarrow 0$, then $p_{X}(m) \rightarrow 1, p_{X}^{\prime}(m) \rightarrow 1$, and $\left|p_{X}(m)-p_{X}^{\prime}(m)\right| \rightarrow 0$.

$$
\begin{aligned}
& \text { If } \max \left(\pi_{2}\left(p_{X}, X\right)-\pi_{2}\left(p_{X}, q_{X}(m)\right), 0\right) \rightarrow 0 \text { and } \\
& \max \left(\pi_{2}\left(p_{X}, X\right)-\pi_{2}\left(p_{X}, q_{X}^{\prime}(m)\right), 0\right) \rightarrow 0 \text {, then } \\
& q_{X}(m) \rightarrow 1, q_{X}^{\prime}(m) \rightarrow 1, \text { and } \mid q_{X}(m)- \\
& q_{X}^{\prime}(m) \mid \rightarrow 0 .
\end{aligned}
$$

(2) When $p_{X}<2 / 3, q_{X}<1 / 3$, if $\max \left(\pi_{1}\left(Y, q_{X}\right)-\right.$ $\left.\pi_{1}\left(p_{X}(m), q_{X}\right), 0\right) \rightarrow 0$ and $\max \left(\pi_{1}\left(Y, q_{X}\right)-\right.$ $\left.\pi_{1}\left(p_{X}^{\prime}(m), q_{X}\right), 0\right) \rightarrow 0$, then $p_{X}(m) \rightarrow 0, p_{X}^{\prime}(m) \rightarrow$ 0 , and $\left|p_{X}(m)-p_{X}^{\prime}(m)\right| \rightarrow 0$.

If $\max \left(\pi_{2}\left(p_{X}, Y\right)-\pi_{2}\left(p_{X}, q_{X}(m)\right), 0\right) \rightarrow 0$ and $\max \left(\pi_{2}\left(p_{X}, Y\right)-\pi_{2}\left(p_{X}, q_{X}^{\prime}(m)\right), 0\right) \rightarrow 0$, then $q_{X}(m) \rightarrow 0, q_{X}^{\prime}(m) \rightarrow 0$, and $\mid q_{X}(m)-$ $q_{X}^{\prime}(m) \mid \rightarrow 0$.

(3) When $p_{X}<2 / 3, q_{X}>1 / 3$, there exist no pair of sequences $\left(p_{X}(m)\right)_{m \geq 1}$ and $\left(q_{X}(m)\right)_{m \geq 1}$ such that $\max \left(\pi_{1}\left(X, q_{X}\right)-\pi_{1}\left(p_{X}(m), q_{X}\right), 0\right) \rightarrow 0$ and $\max \left(\pi_{2}\left(p_{X}, Y\right)-\pi_{2}\left(p_{X}, q_{X}(m)\right), 0\right) \rightarrow 0$.

(4) When $p_{X}>2 / 3, q_{X}<1 / 3$, there exist no pair of sequences $\left(p_{X}(m)\right)_{m \geq 1}$ and $\left(q_{X}(m)\right)_{m \geq 1}$ such that $\max \left(\pi_{1}\left(Y, q_{X}\right)-\pi_{1}\left(p_{X}(m), q_{X}\right), 0\right) \rightarrow 0$ and $\max \left(\pi_{2}\left(p_{X}\right.\right.$, $\left.X)-\pi_{2}\left(p_{X}, q_{X}(m)\right), 0\right) \rightarrow 0$.

(5) When $(2 / 3)-\varepsilon<p_{X}<(2 / 3)+\varepsilon,(1 / 3)-\varepsilon<q_{X}<$ $(1 / 3)+\varepsilon$ with $0<\varepsilon<1 / 3$, if $\max \left(\pi_{1}\left(X, q_{X}\right)-\right.$ $\left.\pi_{1}\left(p_{X}(m), q_{X}\right), 0\right) \rightarrow 0, \max \left(\pi_{1}\left(Y, q_{X}\right)-\pi_{1}\left(p_{X}(m)\right.\right.$, $\left.\left.q_{X}\right), 0\right) \rightarrow 0, \max \left(\pi_{2}\left(p_{X}, X\right)-\pi_{2}\left(p_{X}, q_{X}(m)\right), 0\right) \rightarrow$ 0 , and $\max \left(\pi_{2}\left(p_{X}, Y\right)-\pi_{2}\left(p_{X}, q_{X}(m)\right), 0\right) \rightarrow 0$, then $\left(p_{X}(m), q_{X}(m)\right) \rightarrow(2 / 3,1 / 3)$ for all sequences $\left(p_{X}(m)\right)_{m \geq 1}$ and $\left(q_{X}(m)\right)_{m \geq 1}$.

The payoff functions are sequentially locally nonconstant.

\section{Concluding Remarks}

In this paper, we have presented a constructive procedure to prove the existence of Nash equilibrium in finite strategic games from the viewpoint of constructive mathematics á la Bishop, that is, mathematics based on intuitionistic logic. As a future research program, we are studying the following themes:

(1) an application of the method of this paper to economic theory, in particular, the problem of the existence of an equilibrium in competitive economy with excess demand functions which have the property that is similar to sequential local nonconstancy;

(2) a generalization of the result of this paper to Kakutani's fixed-point theorem for multivalued functions with property of sequential local nonconstancy and its application to economic theory.

For other researches about computability of Nash equilibrium, see [14-18].

\section{Acknowledgment}

This work was supported in part by the Ministry of Education, Science, Sports and Culture of Japan, Grant-in-Aid for Scientific Research (C) no. 20530165.

\section{References}

[1] R. B. Kellogg, T. Y. Li, and J. Yorke, "A constructive proof of Brouwer fixed-point theorem and computational results," SIAM Journal on Numerical Analysis, vol. 13, no. 4, pp. 473483, 1976.

[2] D. Bridges and F. Richman, Varieties of Constructive Mathematics, vol. 97 of London Mathematical Society Lecture Note Series, Cambridge University Press, Cambridge, UK, 1987.

[3] D. van Dalen, "Brouwer's $\mathcal{E}$-fixed point from Sperner's lemma," Theoretical Computer Science, vol. 412, no. 28, pp. 3140-3144, 2011.

[4] V. P. Orevkov, "A constructive mapping of a square onto itself displacing every constructive point," Soviet Mathematics, vol. 4, pp. 1253-1256, 1963.

[5] J. L. Hirst, "Notes on reverse mathematics and Brouwer's fixed point theorem," 2000, http://www.mathsci.appstate.edu/ jlh/ snp/pdfslides/bfp.pdf.

[6] W. Veldman, "Brouwer's approximate fixed-point theorem is equivalent to Brouwer's fan theorem," in Logicism, Intuitionism, and Formalism, S. Lindstrom, E. Palmgren, K. Segerberg, and V. Stoltenberg-Hansen, Eds., vol. 341, pp. 277-299, Springer, Dordrecht, Germany, 2009.

[7] Y. Tanaka, "Constructive proof of Brouwer's fixed point theorem for sequentially locally nonconstant functions," In press, http://arxiv.org/abs/1103.1776.

[8] E. Bishop and D. Bridges, Constructive Analysis, vol. 279, Springer, Berlin, Germany, 1985.

[9] D. Bridges and L. Vîțã, Techniques of Constructive Mathematics, Springer, Berlin, Germany, 2006.

[10] F. E. Su, "Rental harmony: Sperner's lemma in fair division," The American Mathematical Monthly, vol. 106, no. 10, pp. 930942, 1999.

[11] Y. Tanaka, "Equivalence between the existence of an approximate equilibrium in a competitive economy and Sperner's lemma: a constructive analysis," ISRN Applied Mathematics, vol. 2011, Article ID 384625, 15 pages, 2011.

[12] J. Berger, D. Bridges, and P. Schuster, "The fan theorem and unique existence of maxima," Journal of Symbolic Logic, vol. 71, no. 2, pp. 713-720, 2006.

[13] J. Nash, "Non-cooperative games," Annals of Mathematics. Second Series, vol. 54, pp. 286-295, 1951.

[14] S. Takahashi, "The number of pure Nash equilibria in a random game with nondecreasing best responses," Games and Economic Behavior, vol. 63, no. 1, pp. 328-340, 2008.

[15] P. G. Spirakis, "A note on proofs of existence of Nash equilibria in finite strategic games, of two players," Computer Science Review, vol. 3, no. 2, pp. 101-103, 2009.

[16] N. G. Pavlidis, K. E. Parsopoulos, and M. N. Vrahatis, "Computing Nash equilibria through computational intelligence methods," Journal of Computational and Applied Mathematics, vol. 175, no. 1, pp. 113-136, 2005.

[17] W. Stanford, "On the number of pure strategy Nash equilibria in finite common payoffs games," Economics Letters, vol. 62, no. 1, pp. 29-34, 1999. 
[18] V. Conitzer and T. Sandholm, "New complexity results about Nash equilibria," Games and Economic Behavior, vol. 63, no. 2, pp. 621-641, 2008. 


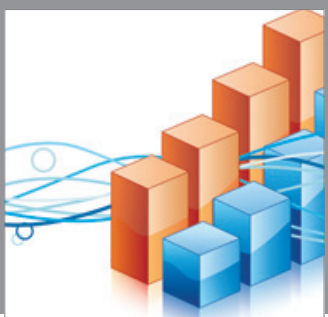

Advances in

Operations Research

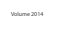

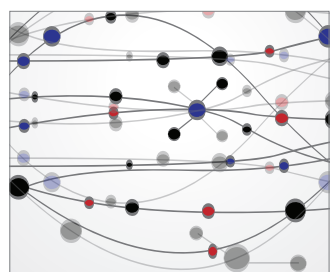

\section{The Scientific} World Journal
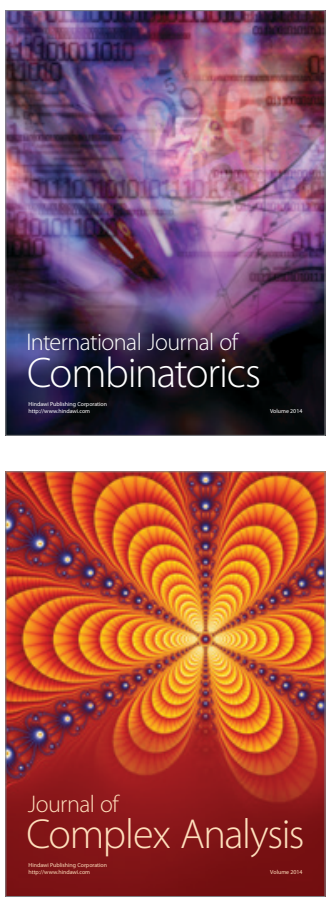

International Journal of

Mathematics and

Mathematical

Sciences
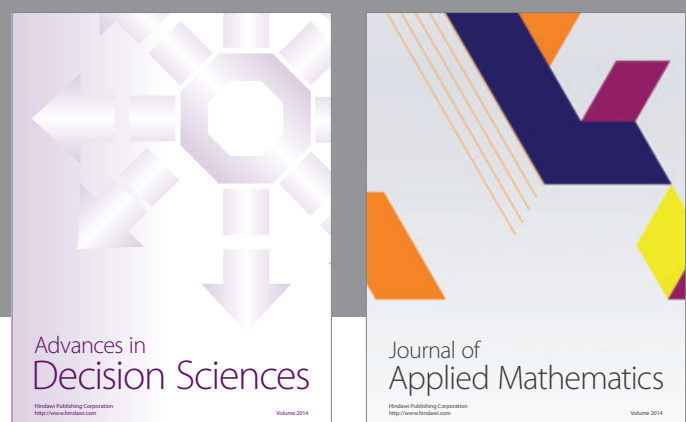

Journal of

Applied Mathematics
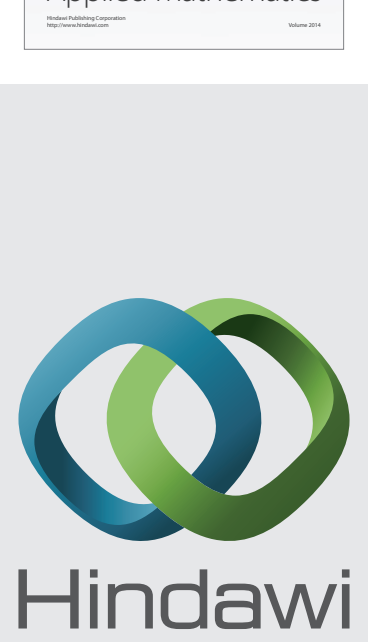

Submit your manuscripts at http://www.hindawi.com
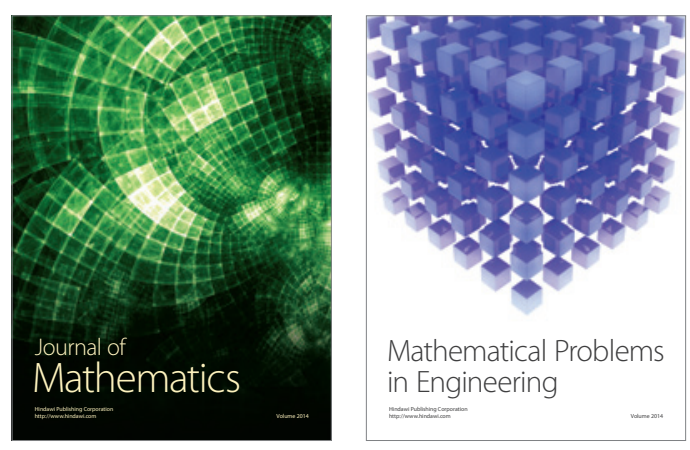

Mathematical Problems in Engineering
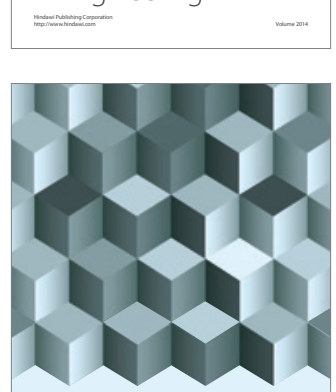

Journal of

Function Spaces
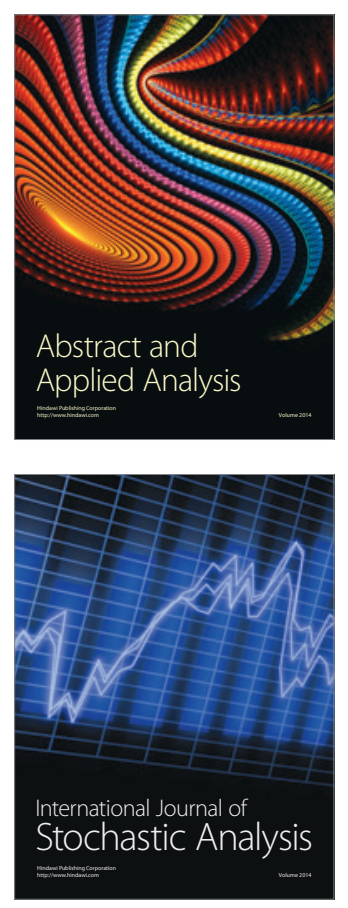

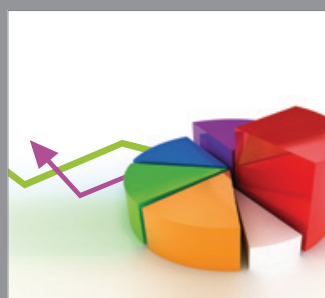

ournal of

Probability and Statistics

Promensencen
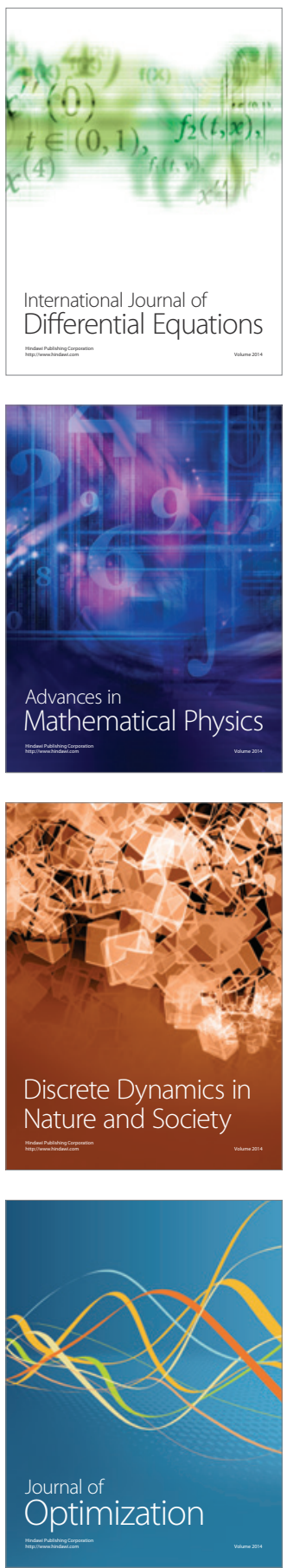